\title{
RETINOL, SUPPLEMENTAL VITAMIN A AND BONE STATUS*
}

\author{
MARYFran R. SOWers ${ }^{\dagger} \dagger$ and Robert B. Wallace ${ }^{2}$ \\ 'Department of Epidemiology, University of Michigan, Ann Arbor, MI 48109 and \\ ${ }^{2}$ Department of Preventive Medicine and Environmental Health, University of Iowa, Iowa City, \\ IA 52242, U.S.A.
}

(Received in revised form 4 October 1989)

\begin{abstract}
Studies of hypervitaminosis A in animals and anecdotal reports of accidental vitamin A poisoning in humans suggest impairment of bone remodeling and increased numbers of fractures. Because of the widespread use of high-dose vitamin A supplements which may produce subclinical hypervitaminosis associated with decreased bone mass and increased risk of fracture, we studied the relationship between current vitamin A supplement use, serum retinol levels, radial bone mass and fracture history in a geographically-defined population of 246 postmenopausal women, 55-80 years of age. More than $36 \%$ of this population used a vitamin A supplement with $8 \%$ of these consuming an amount in excess of 2000 retinol equivalents (RE)/day. Serum retinol was measured using high-pressure liquid chromatography and radial bone mass was measured using single photon absorptiometry. After controlling for age, current estrogen replacement, and current thiazide antihypertensive use, we observed no statistically significant relationship between vitamin A supplement use or serum retinol with radial bone mass or fractures.
\end{abstract}

Vitamin A Retinol Dietary supplements Bone mass Fractures

\section{INTRODUCTION}

Vitamin A can be toxic to a variety of tissues, including bone [1]. Animals fed high doses of the substance acquire systematic changes in growing and mature bones, including selective absorption, cortical thinning and increased numbers of fractures [2]. Clinical studies of the skeletal effects of vitamin $A$, such as in the treatment of dermatologic conditions, have suggested an association with hyperostosis, hypercalcemia and increased bone resorption [3-9].

Because vitamin A supplements are widely available and community consumption has been documented [10-13], investigation of the effects of vitamin $A$ on bone status and health seems to be required. In a population of Japanese-

*Supported by Contract No. NO1-AG-02106.

tAuthor for correspondence.
Americans, dietary vitamin A, obtained by the 24-hour recall method, was positively associated with increased bone density [14]. In a second study, there was a positive relationship between vitamin $A$ and rate of humeral bone loss, and substantial loss in a woman consuming about 4000 retinol equivalents (RE)/day [15]. In a previous cross-sectional study of rural Iowa women, we found that vitamin A intake from diet and supplements was inversely associated with radial bone mass, but the relationship was small and not significant [16].

We are not aware of any clinical or experimental studies of vitamin A and bone mass, and none of the population studies noted above included a biochemical marker of vitamin $\mathrm{A}$ intake. Here we report findings from a different population of Iowa women, exploring the impact of blood vitamin A levels as well as that 
from dietary supplement sources on radial bone mass.

It is the purpose of this paper to undertake the following:

- to test the hypothesis that vitamin A supplementation, or elevated retinol as a biologic marker of vitamin A, may be associated with decreased bone mass in postmenopausal women.

- to test the hypothesis that levels of serum retinol or vitamin A from supplement may be associated with an increase in the report of fracture in postmenopausal women.

\section{METHODS}

All postmenopausal women living in a geographically-defined small rural community in northwest Iowa were invited to participate in a study of bone mass. Those women who were studied reported no disease directly associated with bone mass, had lived in the defined community at least 5 years, were sufficiently ambulatory to climb three stairs, were less than 81 years of age and were of Northern European heritage.

A census of all women in the area was developed by itemizing all residential properties within the city limits and telephoning each property to determine if eligible women were associated with the property. Any property without phone access was visited by a member of the survey team. There were 299 postmenopausal women who were eligible to participate; of those, $263(88 \%)$ consented to interview and bone mass measurement. Both blood sample and bone mass measures were available for $82 \%(n=246)$ of the 299 eligible women. Women who refused participation or blood drawing were more likely to be unmarried and to have fewer years of education. They did not report that they perceived themselves to have less favorable health status than other women their age.

\section{Bone mass measurement and fracture}

Bone mass was measured with a Norland 278 Photon absorptiometer (Norland Corporation; Fort Atkinson, Wisc.) which uses the system originated by Cameron $[17,18]$. Bone mass was measured at a site one-third the distance between the styloid process and the olecranon. Bone mass is expressed as the amount of bone mineral $(\mathrm{g})$, to the bone width $(\mathrm{cm})$.
Our precision, described with the coefficient of variation, is approx. $2 \%$.

Fractures were reported in an interview according to site and year of fracture. For data analysis, fractures were tallied unless associated with a known traumatic event such as vehicular accident. Fracture status was classified three ways. First, as a dichotomous variable, women were categorized as ever having a fracture vs never having a fracture. Second, as a trichotomous variable, women were classified as having fractures of the spine, hip, and wrist vs fractures at other sites vs no reported fractures. Third, the trichotomous variable was restricted to those women who reported their fracture occurring in the 10 years prior to study. Fractures of the spine, hip and wrist are commonly associated with osteoporosis. The fracture classification limited to the previous 10 years was selected to provide measures which may be more contemporary with supplement practice and retinol values.

\section{Assessment of vitamin A}

The biochemical marker selected to represent serum vitamin A was retinol. Vitamin $A$ is a non-specific term for a number of different molecular forms; however, retinol represents an important end-product reflecting enzymatic conversion of $\beta$-carotene, retinyl esters and retinal $[19,20]$. Blood for serum retinol determination was drawn by venipuncture. Serum was drawn off the centrifuged blood, aliquoted, and stored in vials which were frozen at $-20^{\circ} \mathrm{C}$ during field collection up to 63 days and then at $-70^{\circ} \mathrm{C}$ until analysis, which occurred less than 6 months following collection. The serum was protected from light oxidation and was not thawed prior to analysis. Retinol levels were quantified by high pressure liquid chromatography in the vitamin A reference laboratory of Dr James Olson, Iowa State University. Retinol was selected as the most suitable biochemical measure to reflect diet and supplementation practice; it is not intended to reflect dietary consumption within a previous 24 -hour period [19].

Nutrient intake was quantified from two sources: a nutritional supplements interview and 24-hour food recall. The interviewer observed labels of currently used nutrition preparations, including vitamin, mineral and protein supplements, and recorded dose and chemical composition. The participant was asked to recall the 
number, frequency and duration of use of each preparation.

Interviewers were trained to elicit a 24-hour food recall. The interviewer showed color photographs of food to enhance the participant's recall of serving sizes which were reported in common household units. Two different interviewers coded each recall and a nutritionist (MFS) resolved coding differences. Nutrient values were assigned to the coded foods using USDA tape No. 456 which provided nutrient information for 20 nutrients. This food table did not characterize the form of the biologic activity of the vitamin A.

All nutrient values were transformed to common logarithms to normalize their distributions and the normalized values used in data analysis.

\section{Other variables}

Interviewers questioned participants about factors which might affect bone mineralization including demographic information, reproductive history, menopausal history, medical history, activity, sunlight exposure, and use of tobacco and alcohol products. For purposes of analysis, women were defined as postmenopausal if they reported no longer having menses because of surgery or natural history. If menopause was uncertain, a woman was classified as postmenopausal if she had not experienced menses in the 3 months prior to bone mass measurement, was not pregnant or lactating and was in the age range where menopause was likely to occur.

Interviewers observed current medication containers for drug name, dose, and queried respondents about frequency of use. Drugs were coded to be characterized for both intended therapeutic use as well as composition. Women were classified as users of thiazides if their current medications included any thiazide derivative.

Participants were measured for height, weight, midarm circumference, and triceps skinfolds. Humeral muscle area was calculated from these data and used as the variable to reflect body size [21].

Informed consent was obtained in accord with the ethical standards of the Committee on Human Experimentation.

\section{Statistical analyses}

Normality of variable distribution was evaluated with univariate analysis, and those variables whose distribution was highly skewed were either categorized or transformed. Associations between vitamin A levels and bone mass were tested with simple linear and multiple regression analysis [22]. Logistic regression analyses were used to test whether diet vitamin A, supplemental vitamin A or serum retinol was associated with fracture [23].

\section{FINDINGS}

Mean values for serum retinol, supplement vitamin A, and dietary vitamin A are shown in Table 1 according to participant's chronological age, displayed in 5-year age groupings. Because of skewed distributions, dietary vitamin $A$ intake, supplemental vitamin $A$ intake and serum retinol are presented in Figs 1,2 and 3 as median intakes and percentile distributions. Percentile distributions are limited to values between the 10th and 90th percentiles as limited sample sizes generate instability beyond these values. The correlation between vitamin $A$ supplement intake estimate and retinol levels was $0.14, p<0.03$; the correlation between

Table 1. Mean values $( \pm S D)$ of serum retinol, dietary vitamin $A$, and supplement vitamin A among 246 postmenopausal women according to 5-year age groupings

\begin{tabular}{ccccc}
\hline Age group & $n$ & $\begin{array}{c}\text { Retinol } \\
(\mathrm{mmol} / \mathrm{l})\end{array}$ & $\begin{array}{c}\text { Supplement } \\
\text { vitamin A } \\
(\mathrm{RE}) *\end{array}$ & $\begin{array}{c}\text { Dietary } \\
\text { vitamin A } \\
(\mathrm{RE}) *\end{array}$ \\
\hline $40-44$ & 246 & $1.60 \pm 0.54$ & $309 \pm 537$ & $594 \pm 681$ \\
$45-49$ & 10 & $1.38 \pm 0.49$ & $180 \pm 423$ & $475 \pm 793$ \\
$50-54$ & 14 & $1.53 \pm 0.49$ & $214 \pm 425$ & $449 \pm 223$ \\
$55-59$ & 36 & $1.52 \pm 0.45$ & $389 \pm 741$ & $685 \pm 549$ \\
$60-64$ & 28 & $1.60 \pm 0.48$ & $285 \pm 638$ & $379 \pm 493$ \\
$65-69$ & 34 & $1.64 \pm 0.60$ & $329 \pm 522$ & $535 \pm 509$ \\
$70-74$ & 42 & $1.61 \pm 0.54$ & $411 \pm 541$ & $509 \pm 492$ \\
$75-80$ & 46 & $1.63 \pm 0.55$ & $268 \pm 449$ & $655 \pm 739$ \\
\hline
\end{tabular}

*Retinol equivalents. 
retinol and dietary food intake estimate was $0.02, p<0.70$.

\section{Characteristics of supplement users}

In this population of women, $36 \%(n=89)$ reported using a supplement containing vitamin $A$ on a continuing basis within the previous year. Of those supplement users, $54 \%$ had average daily vitamin A supplement intakes of less than $1000 \mathrm{RE} ; 38 \%$ had intakes between 1000 and $2000 \mathrm{RE}$; and $8 \%$ had average daily intakes of vitamin A from supplement greater than 2000 $\mathrm{RE}$, a level which is 2.5 times the Recommended Dietary Allowance [23].

There was a statistically significant relationship in mean serum retinol levels between vitamin A supplement users $(1.69 \pm 0.52 \mathrm{mmol} / \mathrm{l})$ vs non-users $(1.55 \pm 0.52 \mathrm{mmol} / 1, p<0.03)$ (see Table 2).

\section{Potential confounders}

Our previous studies suggested that age, humeral muscle area, thiazide medication use, and perimenopausal estrogen use are significant predictors of bone mass in this population [16]. Another study had suggested that smoking is associated with bone mass [24]. We wanted to

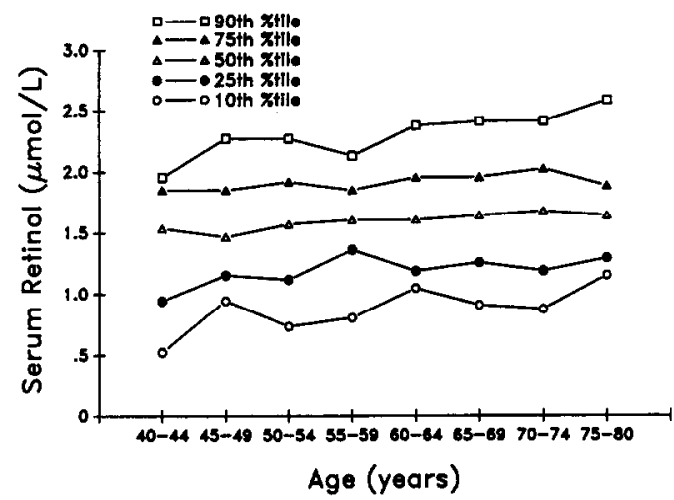

Fig. 1. Percentile distribution of serum retinol of post menopausal women $(n=246)$ representing a geographically defined area.

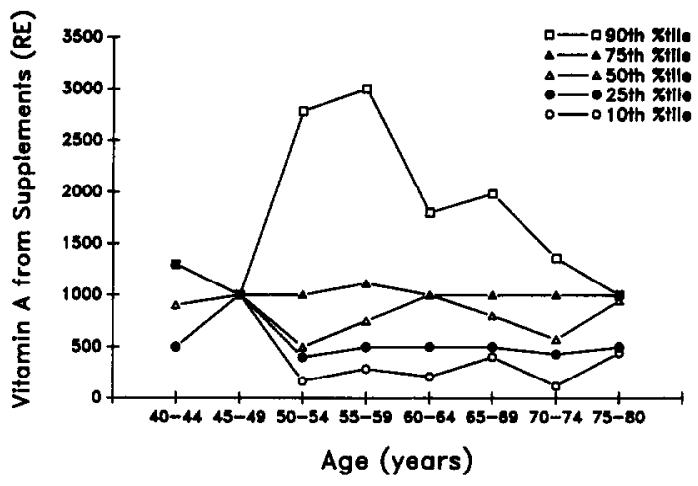

Fig. 2. Percentile distribution of vitamin $A$ intake from supplements in post menopausal women.

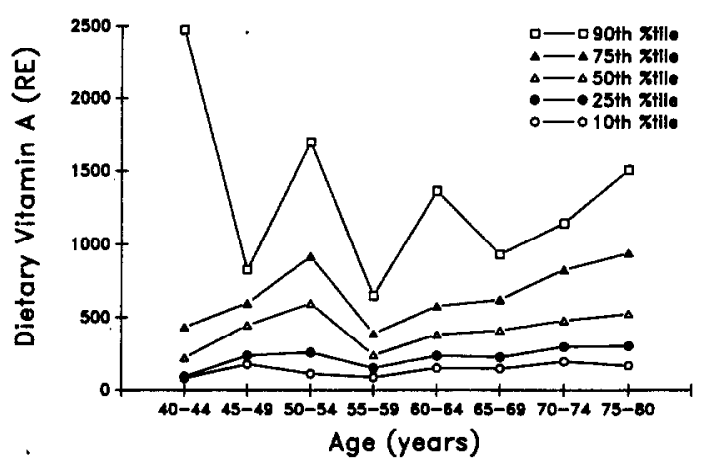

Fig. 3. Percentile distribution of dietary intake of vitamin A as reported by 246 post menopausal women living in a geographically defined area.

determine which factors related to bone mass might also be related to vitamin A supplement use (Table 2). There was no statistically significant difference in potentially confounding factors according to supplement use.

We also examined retinol level relative to age, current thiazide use, current estrogen use, muscle area, and smoking behavior. Serum retinol levels increased with age $(B=0.19$; $r=0.12, p<0.05$ ). Persons who had smoked more than 9000 packs of cigarettes in their lifetime, which represents a median value for smokers in this population, had significantly greater serum retinol levels than those

Table 2. Selected characteristics comparing vitamin A supplement users to nonsupplement users in a geographically defined population of post-menopausal women

\begin{tabular}{lccc}
\hline & $\begin{array}{c}\text { Supplement users } \\
\text { Mean } \pm \text { SD }\end{array}$ & \multicolumn{2}{c}{$\begin{array}{c}\text { Non-users } \\
\text { Mean } \pm \text { SD }\end{array}$} \\
\hline Number & 89 & 157 & \\
Age $(\mathrm{yr})$ & $63.7 \pm 9$ & $62.9 \pm 10$ & $\mathrm{NS}$ \\
Quetelet Index $\left(\mathrm{wt} / \mathrm{ht}^{2}\right)$ & $27.3 \pm 6.2$ & $27.0 \pm 5.7$ & $\mathrm{NS}$ \\
Serum retinol $(\mathrm{mmol} / \mathrm{l})$ & $1.69 \pm 0.52$ & $1.55 \pm 0.52$ & $p<0.03$ \\
Dietary vitamin A (RE) & $679 \pm 756$ & $578 \pm 820$ & $\mathrm{NS}$ \\
Radial bone mass $\left(\mathrm{mg} / \mathrm{cm}^{2}\right)$ & $0.610 \pm 0.11$ & $0.637 \pm 0.12$ & $\mathrm{NS}$ \\
Percent smokers & $26 \%$ & $19 \%$ & $\mathrm{NS}$ \\
Percent current estrogen users & $6 \%$ & $9 \%$ & $\mathrm{NS}$ \\
Percent current thiazide users & $18 \%$ & $25 \%$ & $\mathrm{NS}$ \\
\hline
\end{tabular}


who reported no smoking $(1.90 \pm 0.11 \mathrm{mmol} / \mathrm{l}$ (SEM) vs $1.56 \pm 0.04 \mathrm{mmol} / \mathrm{l}, p<0.0001$ ). After controlling for age, this relationship was still statistically significant $(p<0.01)$.

Thiazide medication use was found to be associated with serum retinol levels. Women currently using thiazide antihypertensive medication had consistently higher mean serum retinol values than did non-users. After adjusting for age, the mean value $( \pm S E M)$ of retinol in women using thiazide was $1.77 \pm 0.07 \mathrm{mmol} / \mathrm{l}$ vs $1.55 \pm 0.04 \mathrm{mmol} / \mathrm{l}$ for women not using thiazide medication $(p<0.008)$. Women who used thiazide medications were more likely to use supplements, though the difference was not statistically significant. Dietary intakes of vitamin A were not different in women who used thiazide medications vs those who did not.

\section{Supplement use, retinol and bone mass}

There was no relationship observed between radial bone mass and supplement intake of vitamin A irrespective of whether the dietary source was supplement alone or diet and supplement amounts combined together, after adjusting for predictors of bone mass including age.

There was no statistically significant relationship between serum retinol and bone mass $(r=-0.04, n=246)$. There was no observed relationship between bone mass and serum retinol level after adjusting for factors associated with bone mass when the population was stratified according to vitamin A supplement use. When retinol values were divided into tertiles and mean bone mass of women in the upper tertile compared with mean bone mass of women in the lower tertile of retinol values, no significant relationship between bone mass was observed when the comparison included adjustment for age, muscle area, and thiazide use.

\section{Supplement use, retinol and fracture}

In this population, there were $98(40 \%)$ women who reported ever having a fracture, 56 $(23 \%)$ who reported an atraumatic fracture of the hip, ribs, spine or wrist postmenopausally, $31(13 \%)$ who reported any fracture in the past 10 years, and $21(8.5 \%)$ who reported fracture of the hip, spine, ribs, or wrist in the past 10 years. We observed no increased probability of observing a fracture associated with the current retinol level irrespective of fracture category. Similarly, we observed no increased probability of observing a fracture associated with current supplement use.

\section{DISCUSSION}

The use of vitamin A supplements is increasingly characteristic of the American population with doses in some instances approaching levels which may be toxic. Up to $60 \%$ of persons are presently using supplement, according to some surveys $[12,13]$. In a study conducted by the Food and Drug Administration in the early 1980s [10], more than 4\% of vitamin A users were taking at least 5 times the Recommended Dietary Allowance of vitamin A [25], a percentage observed in our own studies [11]. This practice is likely to continue as research relating vitamin $\mathrm{A}$ to cancer and skin aging are publicized $[26,27]$ and access to vitamin $A$ is within the domain of personal decision-making. Yet there is limited exploration of bone health in an aging population of women who are using vitamin A supplement preparations in quantities which may approach toxicity.

This study describes the association between serum retinol levels, vitamin A supplement use and indices of bone status in a geographicallydefined population. After controlling for other factors which predict different levels of bone mass, we observed that neither the use of vitamin A supplements in the amount and frequency consumed in this population nor serum retinol are significantly associated with a measure of radial bone mass in postmenopausal women. Furthermore, there was no increased probability of fracture among those women using supplements or with serum retinol level.

The impact of toxic doses of vitamin A on bone mass has been described in three different situations: accidental overdose from supplement use, treatment for skin disorders, and in chronic renal failure. In anecdotal reports of patients being treated for dermatologic disorders, skeletal hyperostosis, increased resorption, hypercalcemia and renal calculi have been observed $[3-9,28]$. In patients with chronic renal problems, plasma concentrations of total vitamin A and retinol-binding protein are increased but the molar ratio of vitamin A to retinol-binding protein are lower than in controls. There are inconsistent observations as to evidence of hypercalcemia and osteodystrophy in renal patients; Cundy et al. and Farrington et al. $[29,30]$ advise caution in prescribing the use of vitamin A supplements to these patients.

In animal and tissue culture studies, hypervitaminosis $A$ leads to reduction in bone mass and characteristics of increased bone resorption [31-38]. Clark and Seawright et al. $[39,40]$ 
described inappropriate architecture and change in morphology of kitten bone, and Clark [41] observed that whereas fractures occur more frequently in the young, rarefaction is more frequently observed in the adult.

It is important to recognize that this study does not test the hypothesis that hypervitaminosis A from vitamin A supplement is negatively related to all aspects of bone health. This study has sufficient sample size and power to test the overall association of supplement with bone mass, but not to test the association of doses exceeding $2000 \mathrm{RE}$ per day, unless differences generated in bone mass are extraordinarily large. Other elements associated with bone health not studied included bone mass at multiple sites, change in bone morphology and joint changes.

This study also does not address the issue of whether dietary vitamin $A$ is associated with levels of bone mass. The intra- and interpersonal variability associated with estimates of vitamin A intake from food is great. To effectively estimate intake requires multiple days of recalls and large sample sizes $[42,43]$. We could not address the issue of vitamin A chemical configuration. Vitamin A represents a group of retinoid and carotenoid compounds, including natural and synthetic forms, which have varying degrees of biologic activity. There was insufficient data to attribute specific biologic activity to the food consumed by this population. Characterizing relationships with less definitively defined products may bias the finding toward the null.

The study involves radial bone mass measurement using single photon densitometry. Though there is much debate as to the need to measure bone at a specific site to characterize fracture risk at that site [44], and bone mass measurement of the spine and hip are now available using dual photon or dual X-ray densitometry, measurement of the radius, in our view, remains an important research tool for population studies. Recently published work has shown, prospectively, that radial bone mass measurements are highly predictive of risk for fracture [45]. Bone mass measurement using the single photon densitometer can be done with greater precision than the dual photon densitometer (approx. 2 vs $4 \%$ ) reducing the sample size needed to identify differences if they exist. There is less exposure in the single photon densitometer as compared with a dual energy densitometer ( $2 \mathrm{mrem}$ vs $5-15 \mathrm{mrem}$ ), desirable for recruiting study populations who are not clinically ill [46].

Bone status evolves as a long-term process whereas this study was able to compare bone status with current supplement practice and single-time biochemical measure. Though this is a limitation, if an association is strong, current practice relationships might be observed, as is the case with estrogen supplementation, and provide motivation for studies of the relationship prospectively.

Though the present study suggested no relationship between supplement use, serum retinol and radial bone mass, an awareness of the possible implications of unsupervised ingestion of large doses of vitamin A should remain a concern of health professionals until additional studies support the observation of no effect.

\section{REFERENCES}

1. Barnicot NA, Datta SP. Vitamin A and bone. In: Bourne GF, Ed. The Biochemistry and Physiology of Bone. New York: Academic Press; 1972: 197-228.

2. Martin TJ, Raisz LG, Eds. Clinical Endocrinology of Calcium Metabolism. New York: Marcel Dekker; 1987.

3. Pittsley RA, Yoder FW. Retinoid Hyperostosis: Skeletal toxicity associated with long-term administration of 13-cis-isoretinoic acid for refractory ichthyosis. N Engl J Med 1983; 308: 1012-1014.

4. Valentic JP, Elias AN, Weinstein GD. Hypercalcemia associated with oral isotretinoin in the treatment of severe acne. JAMA 1983; 250: 1899-1900.

5. Katz CM, Tzagournis M. Chronic adult hypervitaminosis A with hypercalcemia. Metabolism 1972; 21: 1171-1176.

6. Jowsey J, Riggs BL. Bone changes in a patient with hypervitaminosis A. J Clin Endocrinol 1968; 28: 1833-1835.

7. Ragavan VV, Smith JE, Bilezikian JP. Vitamin A toxicity and hypercalcemia. Am J Med Sci 1982; 283: 161-164.

8. Fisher G, Skillern PG. Hypercalcemia due to hypervitaminosis A. JAMA 1974; 227: 1413-1414.

9. Frame B, Jackson CE, Reynolds WA, Umphrey JE Hypercalcemia and skeletal effects in chronic hypervitaminosis A. Ann Intern Med 1974; 80: 44-48.

10. Implications of Vitamin Use. FDA Drug Bull 1983 (Nov.); 13: 26-28.

11. Sowers MFR, Wallace RB. Contribution of water and diet supplements to nutrient intake. J Am Diet Assoc 1986; 86: 1192-1195.

12. Gray GE, Paganini-Hill A, Ross RK. Dietary intake and nutrient supplement use in a Southern California retirement community. Am J Clin Nutr 1983; 38: 122.

13. Schutz HG, Read M, Bendel R, Bhalla VS, Harrill I, Mongale JE, Sheehan ET, Standal BR. Food supplement usage in seven Western states. Am $\mathbf{J}$ Clin Nutr 1982; 36: 897.

14. Yano K, Heilbrun LK, Wasnich RD, Hankin JH, Vogel JM. The relationship between diet and bonemineral content of multiple skeletal sites in elderly Japanese-American men and women living in Hawaii. Am J Clin Nutr 1985; 42: 877-888. 
15. Freudenheim JL, Johnson NE, Smith EL. Relationships between usual nutrient intake and bone-mineral content of women 35-65 years of age: longitudinal and cross-sectional analysis. Am J Clin Nutr 1986; 44: 863-876.

16. Sowers MFR, Wallace RB, Lemke JH. Correlates of mid-radius bone density among postmenopausal women: A community study. Am J Clin Nutr 1985; 41 : $1045-1053$.

17. Cameron JR, Sorenson J. Measurement of bone mineral in vivo: An improved method. Science 1963; 142: $230-232$

18. Cameron JR, Mazess RB, Sorensen JA. Precision and accuracy of bone mineral determination by direct photon absorptiometry. Invest Radiol 1968; 3: 141-150.

19. Garry PJ. Vitamin A. Symposium on Laboratory Assessment of Nutritional Status. Clin Lab Med 1981; 1: 4, 699-711.

20. Arroyave $\mathrm{G}$, Chichester $\mathrm{CO}$, Flores $\mathrm{H}$, Glover J, Mejia LA, Olson JA, Simpson KL, Underwood BA. Biochemical Methodology for the Assessment of Vitamin A Status. International Vitamin A consultative Group; 1982: 1-88.

21. Gray GE, Gray LK. Anthropometric measurements and their interpretation: Principles, practice, problems. J Am Diet Assoc 1980; 77: 524-528.

22. Kleinbaum DG, Kupper LL. Applied Regression Analysis and Other Multivariable Methods. North Scituate, Mass.: Duxbury Press; 1978.

23. Schlesselman JJ. Case-Control Studies: Design, Conduct, Analysis. New York: Oxford University Press; 1982.

24. Daniell HW. Osteoporosis of the slender smoker. Arch Intern Med 1976; 136; 298-304.

25. Committee on Dietary Allowances, Food and Nutrition Board. Recommended Dietary Allowances, 9 th revised edn. Washington, D.C.: National Academy of Sciences; 1980.

26. National Academy of Sciences. Diet, Nutrition and Cancer. Washington, D.C.: National Academy Press; 1982.

27. Weiss JS, Ellis CN, Headington JT, Tincoff T, Hamilton TA, Voorhees JJ. Tropical tretinoin improves photoaged skin: A double-blind vehiclecontrolled study. JAMA 1988; 259: 527-532.

28. Caffey J. Description of the clinical and roentgen manifectatons in seven infants and young children. In: Chronic Poisoning Due to Excess of Vitamin A. New York City: Department of Pediatrics, College of Physicians and Surgeons, Columbia University and the Babies Hospital; 1949.

29. Cundy T, Ernshaw M, Heynen G, Kanis JA. Vitamin A and hyperparathyroid bone disease in uremia. Am $\mathbf{J}$ Clin Nutr 1983; 38: 914-920.

30. Farrington K, Miller P, Varghese Z, Baillod RA, Moorhead JF. Vitamin A toxicity and hypercalcaemia in chronic renal failure. Br Med J 1981; 282: 1999-2002.
31. Fell HB, Mellanby EM. Effects of hypervitaminosis A on feotal mouse bones cultivated in vitro. Br Med $\mathbf{J}$ 1950; 4678: 535-539.

32. Clark I, Smith MR. Effects of hypervitaminosis A and D on skeletal metabolism. J Biol Chem 1964; 239: 1266-1271.

33. Matrajt-Denys $\mathrm{H}$, Tun-Chot $\mathrm{S}$, Bordier $\mathrm{P}$, Hioco $\mathrm{D}$, Clark MB, Pennock J, Doyle FH, Foster GV. Effect of calcitonin on vitamin A-induced changes in bone in the rat. Endocrinology 1971; 88: 129-137.

34. Khogali A. Bone strength and calcium retention of rats in hypervitaminosis-A. Q J Exp Physiol 1966; 51: $120-129$

35. Frankel TL, Seshadri MS, McDowall DB, Cornish CJ. Hypervitaminosis $\mathrm{A}$ and calcium-regulating hormones in the rat. J Nutr 1986; 116: 578-587.

36. Oreffo ROC, Teti A, Triffit JT, Francis MJO, Carano A, Zambonin Zallone A. Effect of vitamin A on bone resorption: evidence for direct stimulation of isolated chicken osteoclasts by retinol and retinoic acid. J Bone Mineral Res 1988; 3: 2: 203-210.

37. Wolke RE, Nielsen SW, Rouseau JE. Bone lesions of hypervitaminosis A in the pig. Am J Vet Res 1968; 29: 1009-1024.

38. Metz AL, Walser MM, Olson WG. The interaction of dietary vitamin $A$ and vitamin $D$ related to skeletal development in the turkey poult. J Nutr 1985; 115 : 929-935.

39. Clark L, Seawright AA, Gartner RJW. Long bone abnormalities in kittens following vitamin $A$ administration. J Comp Pathol 1970; 80: 113-121.

40. Seawright AA, Path MRC, Jrdlicka BE. Pathogenetic factors in tooth loss in young cats on a high daily oral intake of Vitamin A. Aust Vet J 1974; 50: 133-141.

41. Clark I. Mid-diaphyseal bone turnover during and after vitamin A toxicosis in kittens. J Comp Pathol 1971; 81: 365-371.

42. Beaton $\mathrm{GH}$, Milner J, Corey $\mathrm{P}$ et al. Sources of variance in 24-hour dietary recall data: implications for nutrition study design and interpretation. Am $\mathbf{J}$ Clin Nutr 1979; 32: 2546-2559.

43. Liu K. Measurement error and its impact on partial correlation and multiple linear regression analyses. Am J Epidemiol 1988; 127: 864-874.

44. National Center for Health Services Research and Health Care Technology Assessment, Office of Health Technology Assessment. Single Photon Absorptiometry for Measuring Bone Mineral Density, No. 7. U.S. Department of Health and Human Services, Public Health Service; 1986: 1-40.

45. Gardsell $P$, Johnell $O$. Nilsson BE. Predicting fractures in women by using forearm bone disitometry. Calcif Tissue Int 1989; 44: 235-242.

46. Health and Public Policy Committee, American College of Physicians. Radiologic methods to evaluate bone mineral content. Ann Intern Med 1984; 100: 908-911. 